\title{
Germanica
}

\section{La Nuit de Plomb de Hans Henny Jahnn}

Die Nacht aus Blei von Hans Henny Jahnn

\section{André-François Bernard}

\section{(2) OpenEdition}

Journals

Édition électronique

URL : http://journals.openedition.org/germanica/2632

DOI : 10.4000/germanica.2632

ISSN : 2107-0784

\section{Éditeur}

Université de Lille

\section{Édition imprimée}

Date de publication : 31 décembre 1989

Pagination : 145-159

ISSN : 0984-2632

\section{Référence électronique}

André-François Bernard, «La Nuit de Plomb de Hans Henny Jahnn », Germanica [En ligne], 5 | 1989, mis en ligne le 09 février 2015, consulté le 06 octobre 2020. URL : http://journals.openedition.org/ germanica/2632 ; DOI : https://doi.org/10.4000/germanica.2632

Ce document a été généré automatiquement le 6 octobre 2020.

(C) Tous droits réservés 


\title{
La Nuit de Plomb de Hans Henny Jahnn
}

\author{
Die Nacht aus Blei von Hans Henny Jahnn
}

André-François Bernard

1 Hans Henny Jahnn est un auteur peu connu. Il a fallu une récente édition allemande de la plupart de ses œuvres, en onze volumes ${ }^{1}$, pour que le public de la République fédérale d'Allemagne retrouve le souvenir de ce grand écrivain, bien malencontreusement tombé dans l'oubli. Même les éditions de poche, aux catalogues si fréquemment alimentés de nouveaux titres, ne nous proposent qu'une pièce de théâtre de lui, Médée ${ }^{2}$. Un recueil d'extraits existe bien depuis $1984^{3}$, mais la vision que donne le livre de cet homme, dont les champs d'intérêt multiples et l'itinéraire biographique et spirituel ont alimenté une œuvre colossale, reste parcellaire et même partiale. Peu nous agréer en effet que la réflexion des dernières années sur les armes atomiques soit mise en avant, et que cet engagement soit presque interprété comme une anticipation d'une attitude neutraliste qui pourrait être celle d'un adepte des «verts». Il est vrai que le livre, pourtant établi par des spécialistes, veut donner un panorama complet de la pensée de l'auteur, et, en raison même de cette intention, fausse quelque peu l'importance relative des différents blocs d'écriture.

2 Même si les prises de position de cet écrivain ont en maintes circonstances heurté l'opinion, même si certains aspects osés de son œuvre ont suscité la méfiance, il reste que ces discussions, loin de servir l'auteur, le cantonnement plus douloureusement encore dans un recoin de la littérature du Vingtième Siècle, alors qu'il devrait figurer parmi les plus grands.

3 Quelques jalons chronologiques donnent la dimension d'une existence qui fut presque une épopée. Hans Henny Jahnn est né en 1894 à Hambourg, d'une famille de constructeurs de bateaux. Antimilitariste, il fuit son pays en 1915 pour échapper au service militaire et part en Norvège, où il reste jusqu'en 1918. En 1920 il fonde une communauté religieuse libre qui n'aura pas de succès durable et dont il ne restera bientôt plus qu'une maison d'édition. Il s'occupe d'orgues qu'il construit et répare. Et il écrit. Ses livres sont interdits en 1933. En 1934, il émigré au Danemark, à Bornholm, et 
ne rentre à Hambourg qu'en 1950. Là se situe son combat pour des idées politiques, surtout contre le réarmement, mais les circonstances vont vite lui prouver amèrement que mieux vaut pour lui s'abstenir de pareille activité. Il meurt en 1959. Dans l'intervalle, cet écrivain aura été aussi éditeur de partitions baroques, architecte, endocrinologue, cultivateur et éleveur de chevaux.

La Nuit de Plomb, publié en 1955 sans doute la plus achevée de toutes les œuvres de l'auteur, est le seul texte de Jahnn traduit en français. La traduction, assurée par Henri Plard, est complétée par une préface, de lui également, qui apporte de nombreux éléments d'explication ${ }^{4}$.

5 À côté de cette nouvelle, on retiendra le roman de 1929, Perrudja encore tributaire de l'expressionnisme allemand. L'action se situe en Norvège et décrit l'échec d'une tentative utopique de réformer l'humanité. Plus puissante encore est la trilogie Fleuve sans Rives $^{5}$, écrite entre 1934 et 1946, vaste texte sur la création et la solitude de l'homme. Hans Henny Jahnn fut également auteur dramatique ${ }^{6}$, au début et à la fin de sa carrière, mais ses pièces ne sont que des documents qui complètent et corroborent les textes en prose.

6 Thomas Mann a dit dans une lettre à Rolf Italiaander du 22 mars 1954 qu'il admirait tout particulièrement chez Jahnn l'audace de sa conception de l'art. La Nuit de Plomb, par ses thématiques en saillie, est bien une œuvre forte. Elle définit de manière épurée un mal d'être chez un homme jeune qui sort de l'adolescence et montre une éducation intérieure en cours dans la perspective d'un possible achèvement.

7 Le malaise que vient exprimer le livre naît d'une conduite homogène des événements, avec le strict respect d'une règle des trois unités appliquée à la prose : la ville, la nuit, un homme cherche un havre.

8 Les trois phrases qui servent d'ouverture au roman limitent considérablement l'espace psychologique du personnage. Le locuteur invective le personnage: «Je te quitte à présent. Il faut que tu poursuives seul ton chemin. Tu vas explorer cette ville que tu ne connais pas ». L'injonction a le caractère punitif de l'exercice qui vous met en face de votre vérité. Le protagoniste, Mathieu, va devoir apprendre des autres et de la vie à devenir ce qu'il est. Pour conquérir son autonomie, il lui faut cette immersion.

Que l'on compare cette scène du début avec ce qu'aurait pu nous donner sur le même sujet l'Alfred Döblin de Berlin Alexanderplatz, et l'œuvre montre sa spécificité. Point de paysage urbain grouillant de vies humaines qui s'affairent ou déambulent, mais seulement l'individu face à lui-même, la nuit. Le coin de la rue, le large boulevard, les rails de tram, ces détails qui campent le cadre du récit suggèrent plutôt une atmosphère kafkaïenne.

10 Mathieu croit sentir quelqu'un à côté de lui, une présence masculine qui disparaît cependant très vite. La ville lui est étrangère, il n'y connaît personne. Personne dans ce très long boulevard qui semble se prolonger à l'infini. Il aperçoit une fenêtre allumée, seul signe qu'une vie existe, unique but qu'il puisse aussi se donner à lui-même. La fenêtre s'éteint, la lumière des lampadaires commence à faiblir.

Il était né en lui un peu de désir d'être aimé; mais les gouttelettes de la grâce n'étaient pas descendues sur lui. Il errait dans les rues parce que ces vingt-trois années avaient toutes été ternes et sans bonheur ${ }^{7}$.

11 Un homme l'arrête, sorti de dessous un porche. Il lui barre la route et lui donne une carte l'invitant à rentrer dans un cabaret pour voir une femme nommée Elvire. 
12 Cet homme semble avoir percé le secret de Mathieu. Il l'apostrophe. Il ne doit pas continuer son chemin, il a une mission à remplir et a perdu déjà beaucoup trop d'occasions de faire la connaissance des gens, lui dit-il.

13 L'espace de cette ville est existentiel. La narration se compose de scènes-symboles. Hans Henny Jahnn décrit les tourments qui agitent les êtres qui veulent bien se donner la peine de se connaître. Les constructions de l'esprit rejoignent les perceptions de la chair. Mathieu croit sentir le corps d'un garçon contre le sien.

14 Mathieu monte l'escalier et pénètre dans une pièce pompeuse, richement meublée. Des parfums, de la tiédeur, une lumière réfléchie par l'or et les couleurs se déversent sur lui. Un être jeune et joli, un groom en uniforme gris s'adresse à lui. Mathieu touche les cheveux du garçon.

15 La divergence par rapport à une réalité banale et vulgaire est marquée non seulement physiquement par les lèvres peintes du jeune groom (un glissement dans le rêve de la femme à l'homme), mais aussi par le discours que tient ce garçon à Mathieu. La tendresse dont il a fait preuve à son égard est moins gracieuse qu'hier, encore moins qu'avant-hier, et il va recevoir instruction de ce qu'il doit faire.

16 Le discours d'Elvire, sortie par une porte dérobée de la chambre, est celui que tient l'expérience à la jeunesse. La perspective est à la fois psychologique et morale :

Vous voulez fonder vos projets sur une intention et mettre le passé en ordre, de telle manière qu'il prenne un sens. C'est un joli jeu - rien de plus ${ }^{8}$.

17 La scène avec Elvire, qui est un des temps forts du livre, une des articulations de la narration, exclut presque immédiatement une aventure de la chair. Le désir existe cependant en Mathieu, mais il devient vite blessure. Les formes d'Elvire, dégagées entre les plis de la robe, déclenchent en Mathieu plus qu'un trouble affectif ou émotionnel :

Mathieu bondit, blessé d'un vertige aigu comme un poignard. C'était un coup porté en pleine chair, et il sentit les lèvres de la blessure qui bâillaient et le ruissellement du sang.

18 La suite joue de l'opposition baroque entre l'être et le paraître. Mathieu n'a vu que la «forme de la forme ${ }^{10} »$. Le corps de groom est noir, le reste étant de la couleur, « un faux ». « La créature était noire comme le néant, un trou dans la gravitation, existence sans forme ${ }^{11} »$.

19 Le mal d'être a des ramifications profondes. L'imaginaire fonctionne de la manière que nous voyons parce que s'est infiltré en lui le désenchantement.

Nous sommes hommes, de par notre forme ; mais dans notre variante personnelle, un songe, un rideau noir devant nous-mêmes ${ }^{12}$.

20 La réflexion d'auteur s'inscrit dans la fiction élaborée pour le texte : celle d'un jeune homme qui n'a pas encore pris de décision. Elvire dit à Mathieu, peu avant qu'il ne parte, que sa chair est prompte, mais que son esprit sommeille. Le récit, dans sa totalité, s'organise autour de cette divergence entre le réel et les pensées, entre le quotidien et ce monde intérieur parallèle.

21 La description de la pièce prolonge la scène et en forme le commentaire. Le mur en face de Mathieu est de verre, multipliant ainsi la perspective en la dédoublant, cette cloison est un miroir, au delà duquel ne se trouve que l'imaginaire, un second moi.

22 Le sens est clair, la formulation travaillée et poétique, la réflexion intellectuelle sousjacente d'une grande pertinence. 
Les images intérieures se mêlent, se confondent et se superposent. Il croit être Elvire, mais Elvire lui rappelle qu'elle est bien présente, il s'approche, mais alors découvre qu'Elvire est pareille à Grison, qu'elle a le corps noir (le personnage de Médée, dans la pièce du même nom, était également noir). Elvire refuse toute initiative de Mathieu, affirmant qu'il est trop tard, Mathieu lui-même se rendant compte que toutes ses sensations sont en retard.

Mathieu se retrouve dehors, il poursuit son chemin, celui que lui a indiqué le groom. Après quelque temps, un homme l'accoste, s'accroche à lui, ils feront une partie du chemin ensemble, arrivent à un cabaret. Mathieu se reconnaît dans cet homme, cet autre lui rappelle très exactement ce qu'il était huit ou neuf ans en arrière. Il semble " traîner le poids d'un tourment intérieur ${ }^{13}$ ". Mathieu pose au garçon la question s'il est noir ou blanc. Comme dans la scène avec Elvire, le noir est la face cachée de l'être, cette incapacité à exister, cette blessure qui est maintenant reportée sur l'autre, et qui est si profonde qu'elle s'exprime par la chair, le noir du corps est peut-être aussi le corps carbonisé, la mort, au terme de l'amour. Plus grave encore, le noir est aussi la non-existence.

Après cet intermède du café, aux éléments cauchemardesques, plus d'alcool, plus rien à manger, plus d'eau, l'électricité qui faiblit, des propos étranges sur la longueur de la nuit, Mathieu et son compagnon sont dans la rue. Il neige. La neige commence à s'amonceler. L'auteur trouve des mots d'une grande beauté :

Lorsqu'ils s'arrêtèrent une nouvelle fois dans leur marche, ils entendirent le doux tintement des étoiles cristallines, le son des minuscules éclats de glace. Musique troublante par sa monotonie, une gamme qui ne comprenait que quelques intervalles de notes aiguës, mais qui, sous la baguette d'un vent invisible, se déployait en un crescendo et un decrescendo, en des milliers de variations rythmiques. Mélodie inépuisable, peut-être berceuse, qui émoussait la sensibilité, et pourtant bannissait le vrai sommeil, soutenue qu'elle était par les harmoniques d'une inaccessible fatalité - la pétrification du liquide ${ }^{14}$.

Mathieu prend sur son dos l'homme, qui, épuisé, ne peut continuer à marcher. Mathieu est le cheval, l'autre sa monture (on retrouve ici la passion de Hans Henny Jahnn pour les chevaux, le cheval signifie souvent chez Jahnn une exigence de pureté), la couche de neige atteint les genoux de celui qui avance. Étranges propos que ceux de Lautrec (le nom du personnage en allemand est Anders, le traducteur voulant faire passer la notion d'altérité dans le texte français) : il veut retourner dans la cave ; la cave est une demeure pour mourir.

Les sentiments de Mathieu à l'égard du cavalier faiblissent, finissent par s'éteindre entièrement. Une fatigue prend possession de lui et le dépouille de toutes ses facultés.

L'épisode final de la cave, sous le grand collecteur des égouts de la ville se déroule avec la pesanteur du cauchemar. Le mal d'être est accentué dans le sens du morbide.

La longue descente de l'escalier, la marche dans le noir, ce garçon qui n'est autre qu'une perception dédoublée, démultipliée de lui-même, reprend et amplifie les passages précédents du livre. Une volonté nouvelle se fait jour cependant de se confondre avec l'autre, d'inverser le temps, de faire corps avec celui dont il sent très exactement qu'il est celui qui était naguère. Il éprouve alors une plénitude d'être.

Le sentiment d'être arrivé ne le satisfait pas. L'accord avec lui-même se fait dans un système temporel différent. 
Nous ne sommes l'un pour que des ombres, des commentaires inexacts d'un texte quelconque, un quelconque brin de paille qui dérive au fil du temps ${ }^{15}$. Le choix de Mathieu est maintenant clair. Doit-il quitter cette cave, ou bien rester? Faute de la décision énergique de partir, il écoute l'autre : il est le retour de ses quinze ou seize ans, la dégradation vulgaire, le renoncement charnel, la déformation, l'attente déçue, en même temps le désir brûlant. Il est son passé redevenu apparence et chair.

L'équilibre du texte, sa solidité, naît du glissement d'un personnage à l'autre, mais pas seulement de là. La découverte de cette identité présente et de l'identité passée se rejoignent dans la mort. La chambre devient un caveau, les portes se sont refermées. Et par un renversement admirable de situation, en une réciprocité que n'attendait pas le lecteur, c'est maintenant le Mathieu d'aujourd'hui qui s'adresse à celui d'hier, comme pour conjurer sa propre trajectoire de vie et l'on se demande si un choix inverse à celui d'alors ne va pas être fait. Le choix va être confirmé.

Le texte s'enrichit alors d'ouvertures fantastiques: le Mathieu d'hier ne doit pas devenir le Mathieu d'aujourd'hui (ce qui est impossible, l'un étant le dérivé de l'autre), la création a peut-être imaginé ce hasard de créer deux êtres strictement semblables, la commune blessure en témoigne. Quand l'autre montre sa blessure, Mathieu retrouve cette scène d'alors où il avait été agressé par une bande de galopins, puis sauvé in extremis. Pourquoi cette répétition? Sa survie avait-elle donc un sens se demande-t-il ?

4 L'autre raconte l'histoire de cette ville fantastique, de ces gens qui habitent dans cette ville, en sont expulsés en direction du grand champ noir, qui cachent leur corps noir (devenu noir ?) sous des vêtements et se vengent alors de l'étranger, en entaillant ses entrailles chaque jour plus profondément.

Mathieu, obéissant à l'ordre de l'autre, doit ouvrir la trappe du dallage. Une dialectique s'instaure dans leurs rapports. Le blessé veut la chaleur d'un baiser, Mathieu ne veut que procurer de l'aide, l'autre l'accuse de dédaigner son offre. Le dialogue se poursuit à un niveau supérieur. Il faut que la ville noire s'abîme et que le jour se lève. Une dimension monstrueuse est venue chasser l'ordre normal des choses; mais ce dérèglement ne durera plus guère. La réalité, arrachée à ses gonds, doit retrouver sa place. Nous sommes livrés à autrui dans la plus complète des solitudes. Mathieu ressent devant cette chair abimée le vertige de l'angoisse.

Sa main est captive de ce corps. Elle s'en délivrera après un grand tourment.

Mathieu est prisonnier, emmuré, voyant dans cette obscurité une " anti-naissance ${ }^{16}$ ».

«Sa mémoire était dévastée par la folie furieuse du désespoir. Il sentait l'angoisse comme le contact d'une forme ${ }^{17}{ }$.

Une présence physique l'entoure, corps chargé de décrire et ange.

Il entendit avec une précision surnaturelle le fracas du couvercle qui retombait sur le caveau muré18.

La Nuit de Plomb devait être, à l'origine, une scène de rêve à l'intérieur d'un roman, luimême découlant d'un projet de scénario pour un film. Ces divers détours ont affermi et rendu plus incisifs les moments de l'itinéraire décrit, la ville, la nuit, un monde interlope, le tréfonds de la conscience qui se situe difficilement par rapport à ses semblables. Plus que nulle part ailleurs, on sent la contraction de la pensée, qui suggère au lieu d'imposer.

41 On peut lire encore avec intérêt ce livre On est tous rattrapé ${ }^{19}$, à la base de la nouvelle qu'il éclaire en plusieurs points. C'est parce qu'il ne pouvait pas venir à bout de la 
rédaction de son livre que Hans Henny Jahnn a écrit La Nuit de Plomb. Le roman est resté inachevé, mais plusieurs centaines de pages sont là, l'intrigue étant déjà très largement donnée et l'issue préformée. Il semble que nous ayons, en tous cas, et de loin, l'essentiel.

Ce livre parle de l'amitié entre Mathieu, fils d'un grand armateur, et Gari, garçon pauvre qui vit seul avec sa mère. À la suite du naufrage d'un navire de la compagnie, Mathieu est pris à parti et blessé par des garnements qui veulent se venger sur lui de ce que plusieurs des leurs ont péri. Gari s'interpose, mais Mathieu a eu le ventre ouvert d'un coup de couteau. Mathieu se sent désormais lié pour la vie à Gari. Le texte de la nouvelle devait être le cauchemar que fait Mathieu dans sa prison. On l'a accusé d'avoir voulu tuer sa sœur, alors que c'était en fait Gari le meurtrier. Mathieu n'a pas voulu trahir Gari. Dans la nouvelle, la blessure de Mathieu devient celle de son double chez son moi antérieur.

On est tous rattrapé est beaucoup plus direct dans ses affirmation que la nouvelle. Les amitiés particulières des deux adolescents sont très largement décrites, mais le roman ne s'arrête pas à une interprétation monolithique des choses. L'un des deux garçons est riche, frêle, sans aucune attache féminine, est étudiant, l'autre est pauvre, fortement charpenté, a une liaison avec une fille de son âge, qu'il retrouve à chaque escale (il est marin, employé par le père de son ami). Cependant cette opposition est vite dépassée. La somme des différences et des complémentarités crée en gemmellarité qui élève le texte au dessus d'une simple peinture de mœurs. La nouvelle est bien la version définitive d'un texte premier reste en chantier.

Le roman, comme la nouvelle subséquente, décrit une éducation à la vie dans une perspective très étroite d'amours adolescentes. Le tort du roman est de donner une description non réfractée, l'art de la nouvelle est de recentrer le tout dans le cadre d'un rêve devenu cauchemar.

Avec La Nuit de Plomb, le roman d'éducation, tel que nous le propose si souvent la littérature de langue allemande, prend un tour différent. La nouvelle fonctionne à rebours. La démarche narrative, derrière les symboles, les métaphores, les synecdotes, est presque celle de l'investigation psychanalytique. Les différents personnages représentent des strates de la personnalité présente ou passée. Mathieu est un peu chacun des personnages. Chacune des rencontres qu'il fait est une épreuve, et cette épreuve est donnée comme l'exercice d'un être qui veut se libérer de son passé et n'y parvient pas (comme ce qui se passe dans le rêve à la fin de la nuit).

On trouve en toile de fond ce pessimisme foncier que pourrait résumer ce que dit le personnage de Xavier Faltin dans une autre œuvre :

Étudier, pour - pour ne pas devenir plus intelligent, pour n'être pas mieux préparé

à l'existence, mais pour être plus pessimiste ${ }^{20}$.

47 L'histoire de la première édition de La Nuit de Plomb, acceptée, puis refusée, dans sa version illustrée, reflète bien l'historique des malentendus qui entourent l'œuvre. On y a vu une œuvre malsaine, immorale. C'est le sujet même qui rebutait: une relation voilée entre deux garçons, ce thème de la blessure, un troisième garçon appelé l'« ange sombre ".

L'« ange sombre », c'est Gari, qu'invoque Mathieu à la fin du livre ${ }^{21}$. 

pas pour point de départ l'écrivain. On raconterait alors l'histoire de ce facteur d'orgues de grande renommée, instruit et habile, à qui l'on fait appel à Hambourg ou Copenhague ; on aimerait établir plus précisément la biographie de ce passionné de 
sciences qui élève des chevaux à Bornholm. On trouverait un homme aux dons multiples, mais certes pas celui qu'on aurait voulu qu'il fût.

On a taxé l'auteur à l'époque de défenseur de l'homosexualité (a-t-on reproché à Thomas Mann La Mort à Venise!), avant de voir en lui maintenant un ardent défenseur du désarmement. Il est temps de chercher dans l'œuvre cette humanité que l'on concède si facilement à Thomas Mann ou cette sagesse de vie que l'on se plait à attribuer à Hermann Hesse. Il faut enfin reconnaître le talent de cet artiste qui analyse les emplacements complexes de l'être antérieurs à la formulation d'un comportement social policé.

Ce que l'auteur n'a pas réussi à mettre dans sa vie (ses conditions matérielles d'existence furent à plusieurs moments d'une grande indigence) ni dans ses ambitions sociales (sa cité idéale d'Ugrino fut un échec, et il ne fut pas suivi dans le domaine politique), il l'a mis dans ses œuvres : c'est là que l'on rencontre les thèmes positifs de la musique et de la biologie.

Divers destins s'entremêlent pour donner ce grand roman Fleuve sans Rives qui met sur pied d'égalité l'éleveur de chevaux et le génial musicien. La vie est faite de ce passage constant de la vie matérielle à la vie spirituelle, par delà le mal d'être.

L'échec d'une existence en porte-à-faux, attachée à une certaine forme d'idéal se lit tout à fait dans cette période qui entoure la rédaction de La Nuit de Plomb. Le retour en Allemagne, à Hambourg, en 1950, n'a pas l'effet attendu. Même ses amis l'accueillent avec froideur. Il ne jouera pas de rôle dans l'État d'Adenauer. Son idée de vouloir une entente entre l'Est et l'Ouest lui apportera plus de déboires que d'avantages. Ni le "miracle économique ", ni la politique gérée par un parti chrétien ne le satisferont vraiment. On lui fait payer le fait qu'il ne se soit pas vigoureusement opposé au communisme. Qu'il accepte de construire un orgue pour la radio est-allemande, qu'il cherche à se faire publier en R.D.A., le rend suspect aux yeux d'une Allemagne en pleine reconstruction matérielle et morale. Rejeté par l'Ouest, il l'est aussi par l'Est. En juillet 1952, il se rend en R.D.A., mais ne peut faire lever l'interdiction qui pèse sur Fleuve sans Rives. Son voyage à Moscou en 1956 lui vaut les reproches du Sénat hambourgeois.

La Nuit de Plomb est la transcription de cette solitude intérieure qui annule toute action dirigée vers l'extérieur et pétrifie toute relation durable orientée vers l'avenir. L'erreur première est de vouloir intégrer dans le présent, d'abord, harmonieusement, son passé désordonné, sans voir qu'aller simultanément de l'avant permettrait l'épanouissement recherché.

Le mal d'être naît souvent de cette ambiguïté.

\section{NOTES}

1. - Hans Henny Jahnn, Werke in 11 Einzelbänden - Hamburger Ausgabe - Herausgegeben von Uwe Schweikert - Hoffmann und Campe, 1985.

2. - Hans Henny Jahnn, Medea, Universal Bibliothek, Reclam Nr. 8711.1966. 
3. - Hans Henny Jahnn, Lesebuch, Herausgegeben von Uwe Schweikert, Mit einem Nachwort von Helmut Heissenbüttel, Hoff mann und Campe, 1984.

4. - Hans Henny Jahnn, La Nuit de Plomb, traduit de l'allemand par Henri Plard, Éditions du Seuil, 1963, d'après la version Die Nacht aus Blei, europäischer Verlag, Francfort, 1956.

5. - Fluss ohne Ufer (Das Holzschiff / Die Niederschrift des Gustav A nias Horn / Epilog).

6. - Pastor Ephraim Magnus / Die Krönung Richards III / Der Arzt, sein Weib, seinSohn / Der gestohlene Gott / Medea / Strassenecke / Neuer Lübecker Totentanz / Armut, Reichtum, Mensch und Tier / Spur des dunklen Engels / Thomas Chatterton / Die Trümmer des Gewissens.

7. - «Da war ein bisschen Liebesverlangen in ihm erzeugt worden ; aber die Gnade war nicht auf ihn herabgeträufelt. Er zog durch die Strassen, weil diese dreiundzwanzig Jahre so durchschnittlich nicht glücklich gewesen waren» (Die Nacht aus Blei in Hans Henny Jahnn, Werke in 11 Einzelbänden, Hamburger Ausgabe, op. cit., Band 2, S 251) (traduction française, La Nuit de Plomb, op. cit., p. 49).

8. - «Sie wollen Ihrem Vorhaben eine Absicht geben und das Vergangene derart ordnen, dass es sinnvoll wird. Das ist ein hübsches Spiel - nichts weiter» (ibidem, S. 259) (traduction française, ibidem, p. 64).

9. - «Matthieu sprang auf, verwundet von einem Taumel, scharf wie ein Dolch. - Es war ein Stoss, ihm mitten ins Fleisch hinein, und er spürte das Aufklaffen und den Bluterguss» (ibidem, S. 263) (traduction française, ibidem, p. 69).

10. - «Die Form der Form» (ibidem, S. 263) (traduction française, ibidem, p. 70).

11. - «Das Wesen war schwarz wie das Nichts, ein Loch in der Gravitation, Existenz ohne Gestalt» (ibidem, S 264) (traduction française, ibidem, p. 71).

12. - «Wir sind der Gestalt nach Menschen; aber in unserer Abart ein Traum, ein schwarzer Vorhang vor uns selbst» (ibidem, S. 264) (traduction française, ibidem, p. 72).

13. - «Von einem Gram bedrückt» (ibidem, S. 278) (traduction française, ibidem, p. 95).

14. - «Als sie wieder einmal im Ausschreiten innehielten, hörten sie das Klirren der Kristallsterne, den Ton der winzigen Eisscherben. Einen Gesang, unheimlich in seiner einförmigen Skala, die nur wenige hohe Tonschritte enthielt, der aber, durch einen unsichtbaren Wind dirigiert, ein Rlythmisch tausendfach variiertes Crescendo und Decrescendo entfaltete. Eine unausschöpfliche Melodie, gläsern, einschläfernd vielleicht, die Empfindungen abstumpfend, doch den eigentlichen Schlaf fernhaltend durch den Beilaut eines unrührbaren Verhängnisses - der Erstarrung des Flüssigen» (ibidem, S. 288) (traduction française, ibidem, p. 111-112).

15. - «Wir sind für einander doch nur Schatten, Auslegungen, die nicht richtig sind, irgenden Halm, der in der Zeit schwimmt» (ibidem, S 295) (traduction française, ibidem, p. 123).

16. - «Ungeburt» (ibidem, S. 314) (traduction française, ibidem, p. 156).

17. - «Seine Erinnerung war durch den wilden Wahnsinn der Verzweiflung vernichtet. Wie die Berührung durch eine Gestalt fühlte er die Angst», (ibidem, S. 314) (traduction française, ibidem, p. 156).

18. - «Er hörte überdeutlich, dass der Deckel der gemauerten Gruft polternd zufiel» (ibidem, S. 315) (traduction française, ibidem, p. 158).

19. - Jeden ereilt es, commencé en 1951, inachevé.

20. - «Studieren, um - um nicht klüger, nicht gefasster, sondern pessimistischer zu werden» (Epilog, in Fluss ohne Ufer III, Hans Henny Jahnn, Werke in 11 einzelbänden, Hamburger Ausgabe, op. cit., Band 5, S. 61).

21. - «Dunkler Engel» (Die Nacht aus Blei, op. cit., S. 315) (traduction française, op. cit., p. 158).

22. - «Wir brauchen diese Engel als Vorbilder - oder als Ersatzwesen - als Vertreter unserer Sehnsüchte» (Jeden ereilt es, Hans Henny Jahnn, Werke in 11 Einzelbänden, Hamburger Ausgabe, op. cit., Band 2, S. 123). 


\section{RÉSUMÉS}

On ne se dispute pas l'héritage littéraire d'une œuvre tombée dans l'oubli, si vaste soit-elle. L'interprétation d'alors, globale et unilatérale, n'a donc pu être remise en cause : Hans Henny Jahnn a du talent, une immense capacité de production, mais ses qualités d'écriture sont voilées par une approche trop inhabituelle, marginale, solitaire, des problèmes de société et de l'existence. La Nuit de Plomb, texte le plus achevé de l'auteur, bref et particulièrement travaillé, paru en 1955, révèle cette ambiguïté fondamentale. Un homme marche dans la nuit, les lumières de la ville s'éteignent, et voici un être face à lui-même. Par delà une perception des choses désorganisée par un mal d'être profond, c'est le monde troublant des instincts et des pulsions, du tabou, de l'interdit. Mais l'homme ne franchit pas le pas. Il doit donc mourir, emmuré dans le caveau. Seule résonne une adresse à l'« ange ", cette figure tutélaire archétypique qui rebondit d'œuvre en œuvre, et ancre définitivement le récit dans un ensemble narratif plus vaste.

Man streitet sich nicht um das literarische Erbe eines Werkes, das in Vergessenheit geraten ist, so umfangreich es auch sein mag. Die damalige, pauschale und einseitige Interpretation, konnte hier demnach nicht inFrage gestellt werden: Hans Henny Jahnn hat Talent, eine ungeheure Schaffenskraft, aber die Stärken seines Schreibens werden dadurch verdeckt, dass er einen zu ungewohnten und einsamen Zugang eines Aussenseiters zu den Problemen der Gesellschaft und des Daseins hat. Die Nacht aus Bleiy der geschlossenste Text dieses Autors, kurz und besonders sorgfältig durchkomponiert, der im Jahre 1955 erschienen ist, legt von dieser grundlegenden Zweideutigkeit Zeugnis ab. Ein Mann wandert durch die Nacht, die Lichter der Stadt erlöschen, und da ist ein Mensch mit sich selbst konfrontiert. Hinter der Erkenntnis der Dinge, die durch ein tiefes existentielles Unbehagen zerrüttet worden ist, offenbart sich die verwirrende Welt der Instinkte und der Triebe, des Tabus, des Verbotenen. Aber der Mann will die Schwelle nicht überschreiten und muss deshalb in der Gruft eingemauert sterben. Es bleibt nur der Widerhall einer Anrede an den «Engel», an diese archetypische schützende Gestalt, die von Werk zu Werk wieder auftritt und die Erzählung vollends in einen weiteren erzählerischen Zeusammenhang einbindet. 